\title{
Analysis of Pedestrian Lane Change Behavior Spectrum Based on Video Data at Ticket Gate Facilities in Subway Stations
}

\author{
Yong Fang $\mathbb{D}^{D}$, Qi Shi $\mathbb{D}^{D}$, Hua Hu $\left(\mathbb{D}\right.$, Yanxi Hao $\mathbb{D}$, and Zhigang Liu $\mathbb{i}^{\circ}$ \\ School of Urban Rail Transportation, Shanghai University of Engineering Science, Shanghai 201620, China \\ Correspondence should be addressed to Hua Hu; huhua1979@126.com
}

Received 6 August 2021; Revised 13 December 2021; Accepted 6 January 2022; Published 30 January 2022

Academic Editor: Seungjae Lee

Copyright (C) 2022 Yong Fang et al. This is an open access article distributed under the Creative Commons Attribution License, which permits unrestricted use, distribution, and reproduction in any medium, provided the original work is properly cited.

Using motion unit tracking technology, the pedestrian motion parameters were extracted from the monitoring video data at the ticket gate facilities in subway stations and the indicators of the lane change behavior were determined. The pedestrian lane change behavior spectrum of ticket gate facilities in subway stations was constructed from the three elements of types of ticket gate facilities, pedestrian flow, and lane change behavior, and the quartile method was used to determine the upper and lower thresholds of the indicators. The results showed that when the pedestrian flow was $(5,10] \mathrm{ped} / \mathrm{min}$, the average values of displacement, distance, and cumulative side shift distance were the largest and the thresholds were the largest. When the pedestrian flow was $(10,15] \mathrm{ped} / \mathrm{min}$, pedestrians generally adopted a faster walking speed to change lanes. With the increase of the pedestrian flow, the average value of the change in direction of the movement increased and the longitudinal distance at the gatetype ticket gate facilities was greatly affected by the pedestrian flow. The number of lane changes was generally once. The research results can provide a basis for scientifically setting up ticket gate facilities and reducing congestion risks caused by abnormal lane change behavior.

\section{Introduction}

With the construction of smart rail transit, in addition to magnetic cards and noncontact integrated circuit cards, many subway stations have added a mobile scanning method to pass through the ticket gate facilities. Due to diversified ticket checking methods and increasing pedestrian flow, in front of ticket gate facilities in subway stations, pedestrians often change lanes to swipe cards or scan codes when the ticket gate facilities fail. The essence is that when pedestrians are blocked, they take detours to avoid obstacles. These behaviors not only affect the traffic capacity of ticket gate facilities but also increase congestion risks caused by abnormal lane change behavior.

Pedestrian lane change behavior has the characteristics of identifying obstacles and avoiding them in advance [1], and studying the common characteristics of lane change behavior can improve the efficiency of pedestrian passage and the safety of pedestrian movement [2]. Pedestrian lane change behavior spectrum is a data system for comprehensive statistics of the characteristics of lane change behavior. It excludes individual differences of pedestrians (such as gender, age, and whether to carry luggage) and intuitively reflects the common law of pedestrian lane change behavior. By constructing and analyzing the pedestrian lane change behavior spectrum, the thresholds of the indicators can be determined. Through further data analysis, the characteristic indicator of lane change behavior and the threshold that affect the capacity of ticket gate facilities can be extracted to identify abnormal lane change behavior. It provides a theoretical basis for improving the design and setting of ticket gate facilities and reducing pedestrian traffic safety risks.

It is the first time that behavior spectrum theory has been introduced into the study of pedestrian traffic behaviors. Different from the study of the road traffic behavior spectrum, pedestrian traffic behaviors are more complex and diverse, and there are many factors that affect pedestrian traffic behavior characteristics. For example, pedestrians of different genders, ages, and social aspects act a lot on 
pedestrian walking speed and time, modifying their traffic behaviors $[3,4]$. Pedestrians using cell phones or other devices while walking would cause a decrease in attention to the surroundings and walking stability $[5,6]$.

To reveal the common laws of pedestrian traffic behaviors, the research combines individual-group-facility to analyze pedestrian lane change behavior of ticket gate facilities in subway stations as an example and proposes the constituent elements of pedestrian lane change behavior spectrum. Through statistical analysis of the average value, upper and lower threshold range, and standard deviation of the indicators of lane change behavior under different types of ticket gate facilities and pedestrian flow conditions, a quantifiable theoretical system of pedestrian lane change behavior spectrum was formed, which was bottleneck research on the mechanism of interaction between pedestrians and facilities. The construction method of pedestrian traffic behavior spectrum is also applicable to the research of pedestrian behaviors in other public facilities, which is a new step towards the field of pedestrian research. Considering the application of science and technology development and advanced sensors, by constructing different types of pedestrian traffic behavior spectrum, real-time perceptions can be carried out, abnormal traffic behavior can be analyzed, and pedestrian flow intelligence management decisions can be provided.

\section{Literature Review}

Both the evaluation of time to collision (TTC) and pedestrian tracking through different technologies have received widespread attention in recent years [7, 8]. Orsini et al. [9] applied extreme value theory to road safety and analyzed a case of dealing with motorway rear-end collision risk. Tesoriere et al. [10] recorded the passenger volumes of the multinode in different periods by photos or videos and used the social force model method to analyze airport terminals with huge amounts of passengers passing by every hour. Wei et al. [11] used Hadoop architecture to analyze and store the data, and the continuous adjacent videos were spliced and stored. And then, pedestrian trajectories and basic pedestrian motion parameters in a two-dimensional plane were extracted. Ismail et al. [12] described a novel technique for conducting road safety analysis using an automated video analysis system based on four steps: data collection, camera calibration, extraction of road user tracks, and calculation of conflict indicators, and using computer vision techniques to automatically analyze pedestrian-vehicle conflicts proved to be feasible. Basbas et al. [13] obtained indicators such as the length of the pedestrian street, effective width, and average pedestrian walking speed through field measurements and used the Viswalk pedestrian traffic simulation software to evaluate the current status of the pedestrian street. Gruden et al. $[14,15]$ obtained real-world information by video footages shot by cameras, set in order to cover the whole area of interest, and elaborated real-world information via a semiautomatic detection and tracking software to achieve trajectories and then constructed different pedestrian microscopic simulation models based on real data.
At present, there are little researches on the characteristics of pedestrian traffic behaviors of ticket gate facilities. Daamen and Hoogendoorn [16] developed the microscopic simulation model software NOMAD that reflected the individual characteristics of pedestrians and analyzed the influence of the ticket gate facilities on the pedestrian congestion level, average travel time, delay, and other indicators. $\mathrm{Wu}$ [17] proposed that pedestrians must complete ticket checking and passing behavior at the ticket gate facilities and analyzed the actual capacity with indicators such as the number of people who did not know how to swipe the card and the average number of passers per minute. Shao [18] analyzed the actual capacity of the ticket gate facilities with indicators such as the walking deviation of individual pedestrians, the stops of pedestrians, and the waiting time of pedestrians. It can be found that there are related indicators in the existing studies when pedestrians cannot pass smoothly at the ticket gate facilities, but there is no detailed analysis of pedestrian lane change behavior.

The traffic behavior spectrum is considered to be a basic data system that comprehensively records the behavioral commonality of pedestrians, motorized vehicle drivers, and nonmotorized vehicle drivers in the traffic process [19]. This data system includes both microscopic behaviors such as operation behaviors, driving behaviors, and pedestrian behaviors, but also macroscopic behaviors, such as travel behaviors. There are not many existing pieces of researches on the traffic behavior spectrum [20-22]. He et al. [23] used indicators such as speed headway, headway distance, relative speed, and TTC as the constituent elements to construct carfollowing behavior spectrums of drivers in 15 typical carfollowing driving scenarios. Based on the car-following behavior spectrum, Wang et al. [24] extracted vehicle kinematics indicators such as acceleration and headway distance and driver manipulation indicators such as accelerator pedal force and steering wheel angle to determine 32 risk characteristic indicators. According to the risk characteristic indicators, three car-following risk state indicator thresholds, namely, the reciprocal collision time, lateral swing coefficient, and speed variation coefficient, were determined, and the machine learning prediction model was used to predict the car-following risk state. Chang et al. [25] established a map of bad vehicle behaviors under different traffic flows by analyzing the characteristics of vehicle behaviors on urban roads and the impact of microscopic vehicle behaviors on the traffic flow. Wang et al. [26] proposed a method for determining the threshold of bad driving behaviors and the method for calculating the characteristic value of bad driving behavior spectrum based on large sample statistical distribution, which could be applied to real-time discrimination.

\section{Method}

3.1. Constituent Elements of Pedestrian Lane Change Behavior Spectrum of Ticket Gate Facilities in Subway Stations. In the process of walking, pedestrians will adjust their personal traffic behaviors according to their own motion characteristics and the surroundings and move in the desired direction. Due to the susceptibility to the movement of 
surrounding pedestrians, interactive behaviors will occur between pedestrians and pedestrians. These behaviors (such as avoidance behaviors and overtaking behaviors) easily disturb the stability of pedestrian group movement, resulting in risks such as crowding, collisions, and trampling. Pedestrians are also susceptible to the types of facilities and the spatial scale of facilities. They adjust their traffic behaviors through visual and auditory environment cognition. The interaction between pedestrian groups and facilities affects the capacity of facilities and the ability of pedestrians to safely evacuate. The above analysis shows that individual pedestrians, groups, and facilities are interrelated and influence each other. Combining individual-group-facility to carry out research can reveal the common laws of individual pedestrian traffic behaviors, and the pedestrian traffic behavior spectrum expresses a basic data system of pedestrian traffic behavior commonality during walking. Therefore, the set of constituent elements proposed in the research is shown in Figure 1. To construct a pedestrian traffic behavior spectrum, individual elements, group elements, and facility elements need to be considered.

According to the above description, the pedestrian lane change behavior spectrum of ticket gate facilities in subway stations should include the following types of elements.

Types of ticket gate facilities: the most common types of ticket gate facilities are gate-type and three-bar-type ticket gate facilities.

Pedestrian group: the pedestrian flow was used to reflect the characteristics of the pedestrian group, which was the number of pedestrians passing through the ticket gate facility per unit time. The study found that there was a big difference between the actual capacity of ticket gate facilities and the designed capacity [27]. According to the on-site observation of pedestrian flow data per minute, the maximum actual pedestrian flow was $15 \mathrm{ped} / \mathrm{min}$. When the pedestrian flow was $[0,5]$ ped/min, pedestrians could freely choose the ticket gate facilities and walking speed to pass, and the walking was basically not affected by other pedestrians in the pedestrian flow. The pedestrian flow was in a free flow. When the pedestrian flow was $(5,10] \mathrm{ped} / \mathrm{min}$, pedestrians were greatly interfered by other pedestrians in the pedestrian flow, and their walking speed was limited to a certain extent. The pedestrian flow was in a stable flow, which had a sense of congestion. When the pedestrian flow was $(10,15]$ ped/ min, pedestrians were more severely interfered by other pedestrians in the pedestrian flow. The pedestrian flow was in an unstable flow state. When approaching the upper limit, the pedestrian flow per minute at the ticket gate facility reached the maximum, and pedestrians had no room for free choice of walking speed. According to the different pedestrian flow states found in actual observations, this research divided the pedestrian flow into $[0,5],(5,10]$, and $(10,15] \mathrm{ped} / \mathrm{min}$ for research.

Individual lane change behavior: related indicators of the movement characteristics of individual pedestrians were used when the behavior occurred. Generally, it would include indicators such as speed, displacement, direction, and distance.
3.2. The Indicators of Lane Change Behavior. When pedestrians cannot pass through the ticket gate facilities in the subway station smoothly, the pedestrians will adjust the walking path according to the location of the surrounding pedestrians and ticket gate facilities and adopt a detour behavior. Regarding the research on pedestrians' lane change behavior by others [1] and drivers' lane change behavior in road traffic $[19,28,29]$, pedestrians or vehicles could be regarded as motion units. This research believed that pedestrian lane change behavior could be characterized by the pedestrian's own motion state, the relative position of the ticket gate facilities, and the frequency of lane change.

The pedestrian's own motion state mainly started with the pedestrian's motion trajectory and the speed of the lane change behavior. The indicators were displacement $|\vec{s}|$, distance $S$, cumulative side shift distance $D_{S}$, change in direction of the movement $\Delta \theta$, and average speed $\bar{V}$.

The relative position of the ticket gate facility could be characterized by the longitudinal distance $D_{V}$, which could determine the distance for pedestrians to identify obstacles and avoid them in advance.

The frequency of lane changes could be characterized by the number of lane changes. Counting the number of lane changes of pedestrians at the ticket gate facilities could find the group law of lane change frequency and explore the internal connection with the safety of pedestrian groups and the number of ticket gate facilities. The indicators of pedestrian lane change behavior at the ticket gate facilities are shown in Figure 2.

Displacement $|\vec{s}|$ referred to the linear distance between the start and the end of pedestrian lane change behavior, as shown in the following equation:

$$
|\vec{s}|=\sqrt{\left(x_{n}-x_{1}\right)^{2}+\left(y_{n}-y_{1}\right)^{2}},
$$

where $\left(x_{1}, y_{1}\right)$ was the start position of the lane change behavior and $\left(x_{n}, y_{n}\right)$ was the end position of the lane change behavior.

Distance $S$ referred to the total distance travelled by pedestrians, as shown in the following equation:

$$
S=\sum_{i=1}^{n} S_{i}, \quad i=1,2, \ldots, n,
$$

where $S_{i}$ was the distance travelled by pedestrians at a certain time.

Average speed $\bar{V}$ referred to the ratio of pedestrian distance to time, as shown in the following equation:

$$
\bar{V}=\frac{S}{t_{n}-t_{1}},
$$

where $t_{1}$ was the start moment of the lane change behavior and $t_{n}$ was the end moment of the lane change behavior.

Change in direction of the movement $\Delta \theta$ referred to the angle of change between the expected moving direction and the actual moving direction at the beginning of the pedestrian lane change behavior, as shown in the following equation: 


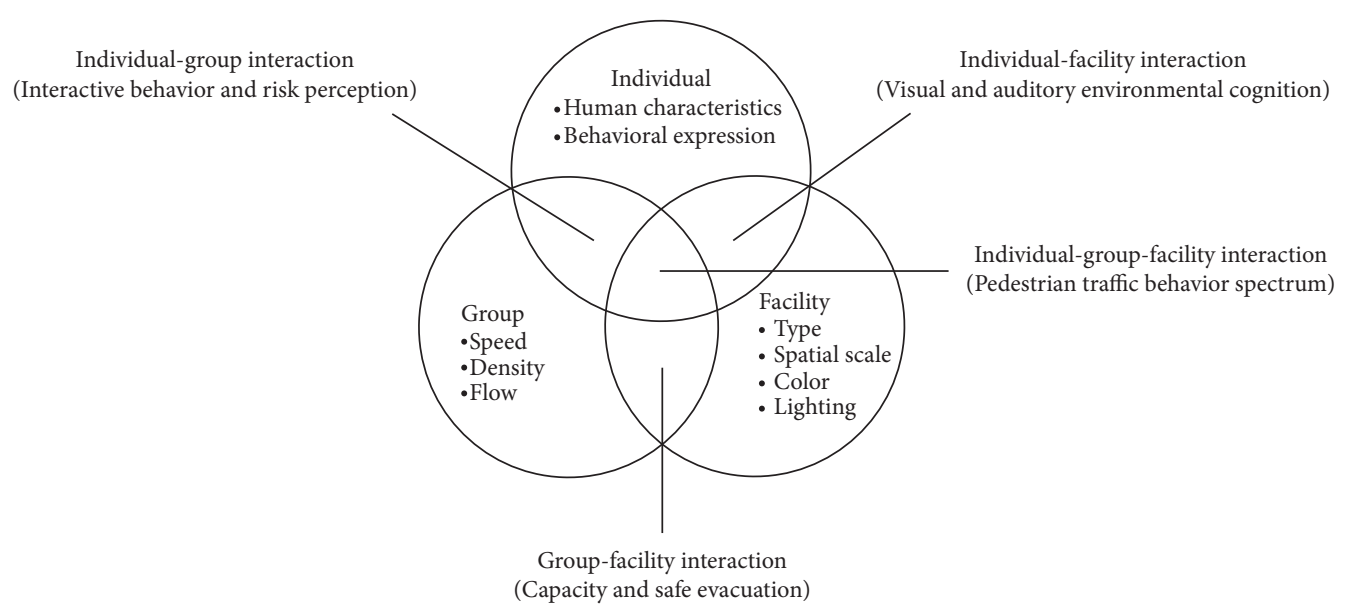

Figure 1: Constituent elements of the pedestrian traffic behavior spectrum.

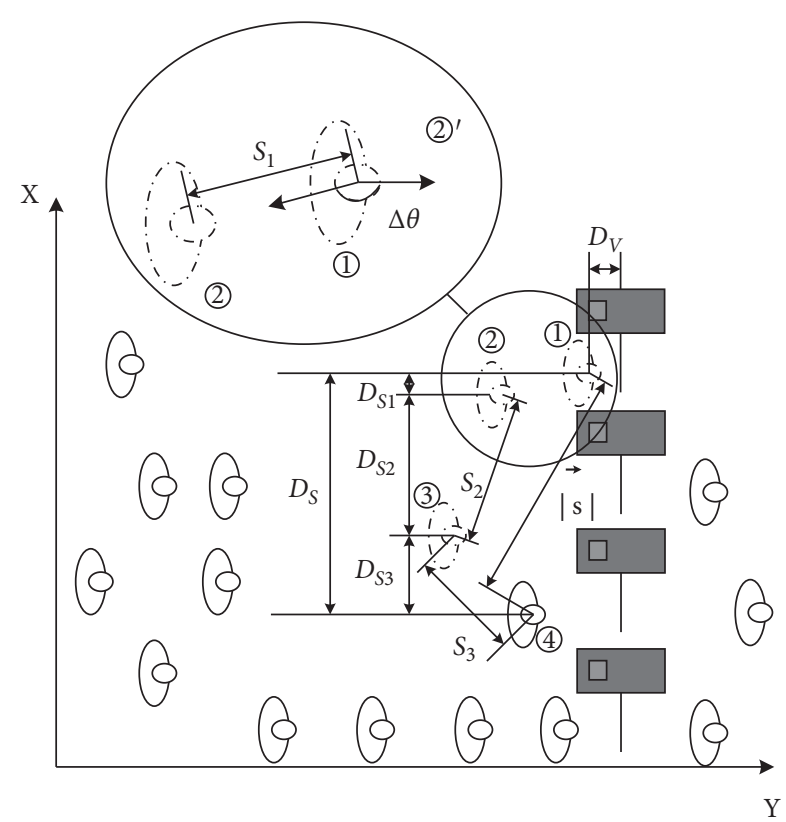

FIgURE 2: The indicators of pedestrian lane change behavior at the ticket gate facilities.

$$
\theta=\left|\left(\tan ^{-1}\left(\frac{y_{2}-y_{1}}{x_{2}-x_{1}}\right)-\tan ^{-1}\left(\frac{y_{2}^{\prime}-y_{1}}{x_{2}^{\prime}-x_{1}}\right)\right) \times \frac{180}{\pi}\right|,
$$

where $\left(x_{2}, y_{2}\right)$ was the actual moving position and $\left(x_{2}^{\prime} y_{2}^{\prime}\right)$ was the expected moving position, and it was assumed that pedestrians expected to move to the ticket gate facility directly ahead.

Longitudinal distance $D_{V}$ referred to the longitudinal distance between the start point of pedestrian lane change behavior and the center of the ticket gate facility, as shown in the following equation:

$$
D_{V}=\left|y_{1}-y_{0}\right|
$$

where $y_{1}$ was the ordinate of the start position of the lane change behavior and $y_{0}$ was the ordinate of the center position of the ticket gate facility.
Cumulative side shift distance $D_{s}$ referred to the cumulative lateral distance between the start and end points of pedestrian lane change behavior, as shown in the following equation:

$$
\begin{aligned}
D_{s} & =\sum_{i=1}^{m} D_{S i} \\
& =\sum_{i=1}^{m}\left|x_{i}-x_{i-1}\right|,
\end{aligned}
$$

where $D_{s i}$ was a single-step lateral distance of the pedestrian.

The number of lane changes $k$ referred to the number of times that a single pedestrian changed lane when he completed the lane change behavior.

\subsection{Construction Method of Pedestrian Lane Change Behavior} Spectrum. When the indicator value of lane change behavior is significantly smaller or larger, it will have a significant impact on the actual capacity of the ticket gate facility and the congestion risk. Therefore, it could be assumed that the indicator value of lane change behavior should be concentrated in a reasonable range, and far away this interval was the abnormal value. The interquartile method was common in outlier detection, which could be used to calculate the threshold of various distributed data [30]. In this research, the quartile method was used as a method to determine the threshold value of indicators of lane change behavior to obtain the upper and lower threshold range, which could be further used as a basis for distinguishing abnormal lane change behavior.

The formula for the threshold $M_{i}^{*}$ of the indicator of lane change behavior was shown in the following equation:

$$
M_{i}^{*}=\left[\max \left(0, Q_{i 1}-1.5 I Q R_{i}\right), Q_{i 3}+1.5 I Q R_{i}\right],
$$

where $Q_{i 1}$ was the lower quartile of the sample distribution of the indicator $i$, and it was generally the 25th quantile, and $Q_{i 3}$ was the upper quartile of the sample distribution of the indicator $i$, and it was generally the 75 th quantile. $\mathrm{IQR}_{i}$ was interquartile difference. Since the indicators of lane change 
behavior were all positive numbers, when $Q_{i 1}-1.5 \mathrm{IQR}_{i}<0$, the lower limit of the threshold should be 0 .

Under different pedestrian flow, the average value, the upper and lower threshold, and fluctuation range of the indicators of lane change behavior at each type of ticket gate facilities were collected to construct a pedestrian lane change behavior spectrum of ticket gate facilities in subway stations, as shown in Table 1.

\section{Experiment}

The data was collected from the monitoring videos of the ticket gate facilities in multiple subway stations in Shanghai on a weekday (5 days). The number of ticket gate facilities in each subway station was 5 , of which the number of gate-type ticket gate facilities was 2 . The number of three-bar-type ticket gate facilities was 3 . The collection time was 16:00-18: 00 during the afternoon peak time. The video resolution was 704 pixels $\times 576$ pixels, and the frame rate was 24 frames $\cdot \mathrm{s}^{-1}$. Part of the pedestrians that were difficult to identify or the identification error of whom was significantly larger were eliminated. After screening, there were 477 sample data sets of pedestrian lane change behaviors used in this research.

Since the angle of the monitoring video camera was not completely perpendicular to the ground, and the video image shooting angle was inclined, the method of extracting pedestrian behavior data from the camera's top view could not be used [31]. Adobe After Effects software was used to automatically track pedestrians. The pedestrian's face was used as the tracking target, and the pixel coordinates of the tracking target were recorded every frame. Figure 3(a) shows the tracking target pixel coordinate extraction. Then the camera was calibrated to obtain the camera internal parameter matrix, rotation matrix, translation vector, and other parameters, as shown in Figure 3(b). According to the method provided by Zhou [32], the two-dimensional pixel coordinates of the tracking target were converted into realworld coordinates. Through the change of pedestrian position coordinates in the video screen within a certain time, the indicators of lane change behavior could be calculated.

\section{Results}

\subsection{Analysis of the Indicators of Lane Change Behavior}

5.1.1. Displacement. Figure 4 shows the distribution of displacement of pedestrian lane change behavior. At gatetype ticket gate facilities, when the pedestrian flow was $[0,5]$ ped/min, the lower quartile $Q_{|\vec{s}| 1}$ was $0.91 \mathrm{~m}$, the upper quartile $Q_{|\vec{s}| 3}$ was $1.64 \mathrm{~m}$, then the interquartile difference $\mathrm{IQR}_{|\vec{s}|}$ was $0.73 \mathrm{~m}$, and the threshold $M_{|\vec{s}|}^{*}$ was $(0,2.74] \mathrm{m}$. Similarly, when the pedestrian flow was $(5,10]$ and $(10,15]$ ped/min, the threshold $M_{|\overrightarrow{\mid}|}^{*}$ was $(0,4.38] \mathrm{m}$ and $(0,3.33]$ $\mathrm{m}$. At three-bar-type ticket gate facilities, when the pedestrian flow was $[0,5],(5,10]$, and $(10,15] \mathrm{ped} / \mathrm{min}$, the threshold $M_{\rightarrow}^{*}$ was $(0,4.42] \mathrm{m},(0,5.58] \mathrm{m}$, and $(0,3.94] \mathrm{m}$.

At different types of ticket gate facilities, when the pedestrian flow was $(5,10] \mathrm{ped} / \mathrm{min}$, the average value of displacement $|\vec{s}|$ was the largest and the threshold $M_{\vec{s}}^{*}$ was the largest. As the pedestrian flow increased, the $\overrightarrow{\mathrm{s}}$ destrian movement space decreased, and the threshold $M_{|\overrightarrow{\boldsymbol{s}}|}^{*}$ decreased significantly.

5.1.2. Distance. Figure 5 shows the distribution of distance of pedestrian lane change behavior. At gate-type ticket gate facilities, when the pedestrian flow was $[0,5],(5,10]$, and $(10$, 15] ped/min, the threshold $M_{S}^{*}$ was $(0,4.21] \mathrm{m},(0,4.35] \mathrm{m}$, and $(0,3.33] \mathrm{m}$. At three-bar-type ticket gate facilities, when the pedestrian flow was $[0,5],(5,10]$, and $(10,15] \mathrm{ped} / \mathrm{min}$, the threshold $M_{S}^{*}$ was $(0,4.42] \mathrm{m},(0,5.33] \mathrm{m}$, and $(0,4.48] \mathrm{m}$.

The three-bar-type ticket gate facilities had a greater impact on the interquartile range of distance $S$, while the gate-type ticket gate facilities had a smaller impact. At different types of ticket gate facilities, when the pedestrian flow was $(5,10] \mathrm{ped} / \mathrm{min}$, the average value of distance $S$ was the largest, and the threshold $M_{S}^{*}$ was the largest. As the pedestrian flow increased, the pedestrian movement space decreased, and the threshold $M_{S}^{*}$ decreased significantly.

5.1.3. Cumulative Side Shift Distance. Figure 6 shows the distribution of cumulative side shift distance of pedestrian lane change behavior. At gate-type ticket gate facilities, when the pedestrian flow was $[0,5],(5,10]$, and $(10,15] \mathrm{ped} / \mathrm{min}$, the threshold $M_{D_{s}}^{*}$ was $(0,3.29] \mathrm{m},(0,3.28] \mathrm{m}$, and $(0,3.03]$ $\mathrm{m}$. At three-bar-type ticket gate facilities, when the pedestrian flow was $[0,5],(5,10]$, and $(10,15] \mathrm{ped} / \mathrm{min}$, the threshold $M_{D_{s}}^{*}$ was $(0,3.00] \mathrm{m},(0,4.27] \mathrm{m}$, and $(0,3.77] \mathrm{m}$.

At different types of ticket gate facilities, when the pedestrian flow was $(5,10] \mathrm{ped} / \mathrm{min}$, the average value of cumulative side shift distance $D_{S}$ was the largest and the threshold $M_{D_{S}}^{*}$ was the largest. As the pedestrian flow increased, the pedestrian movement space decreased, and the threshold $M_{D_{S}}^{*}$ decreased.

5.1.4. Change in Direction of the Movement. Figure 7 shows the distribution of change in direction of the movement of pedestrian lane change behavior. At gate-type ticket gate facilities, when the pedestrian flow was $[0,5],(5,10]$, and $(10$, 15] ped/min, the threshold $M_{\Delta \theta}^{*}$ was $\left[21.03^{\circ}, 126.55^{\circ}\right],\left(0^{\circ}\right.$, $\left.184.27^{\circ}\right]$, and $\left(0^{\circ}, 194.10^{\circ}\right]$. At three-bar-type ticket gate facilities, when the pedestrian flow was $[0,5],(5,10]$, and $(10$, 15] ped/min, the threshold $M_{\Delta \theta}^{*}$ was $\left[41.56^{\circ}, 101.20^{\circ}\right],\left[9.28^{\circ}\right.$, $\left.160.32^{\circ}\right]$, and $\left[51.53^{\circ}, 172.29^{\circ}\right]$.

At different types of ticket gate facilities, as pedestrian flow increased, the average value of change in direction of the movement $\Delta \theta$ increased, and the upper quartile $Q_{\Delta \theta 3}$ increased significantly, indicating that the angle of pedestrian lane change behavior was affected by the number of pedestrians at adjacent ticket gate facilities.

5.1.5. Average Speed. Figure 8 shows the distribution of average speed of pedestrian lane change behavior. At gatetype ticket gate facilities, when the pedestrian flow was $[0,5]$, $(5,10]$, and $(10,15] \mathrm{ped} / \mathrm{min}$, the threshold $M_{\bar{V}}^{*}$ was $(0,1.81]$ $\mathrm{m} / \mathrm{s},[0.01,1.29] \mathrm{m} / \mathrm{s}$, and $[0.58,1.42] \mathrm{m} / \mathrm{s}$. At three-bar-type 
TABLE 1: Example of pedestrian lane change behavior spectrum of ticket gate facilities in subway stations.

Types of ticket gate facilities

Pedestrian flow

Displacement $|\vec{s}|$

Distance $S$

Average speed $\bar{V}$

Change in direction of the movement $\Delta \theta$

Longitudinal distance $D_{V}$

Cumulative side shift distance $D_{S}$

Number of lane changes $k$

Gate-type, three-bar-type

$[0,5],(5,10],(10,15]$

Average value, upper limit, standard deviation

Average value, upper limit, standard deviation

Average value, upper limit, lower limit, standard deviation Average value, upper limit, lower limit, standard deviation Average value, upper limit, lower limit, standard deviation Average value, upper limit, standard deviation Average value, upper limit, lower limit, standard deviation

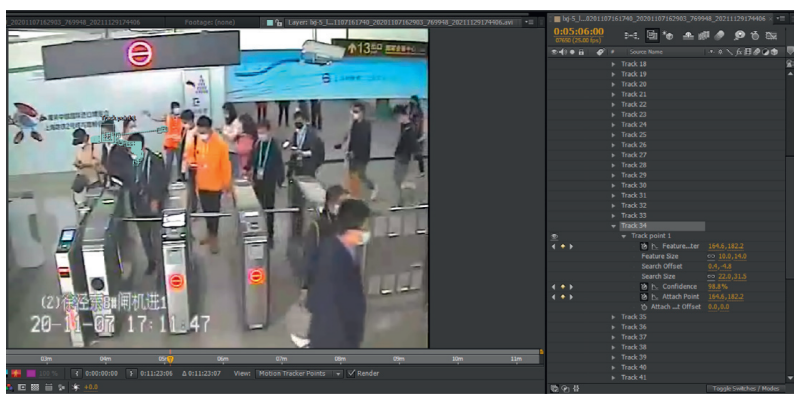

(a)
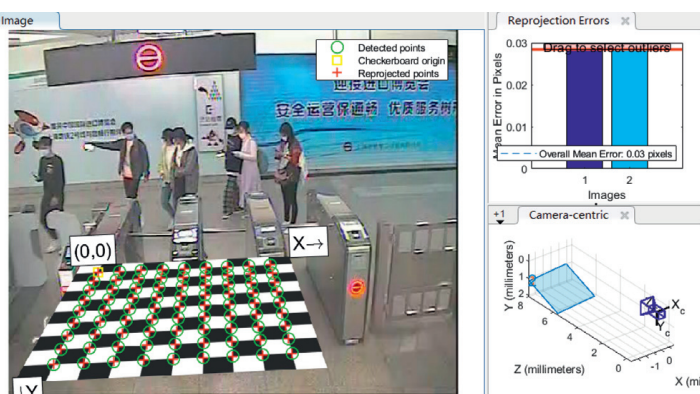

(b)

Figure 3: The process of data collection. (a) Tracking target pixel coordinate extraction. (b) Camera calibration.

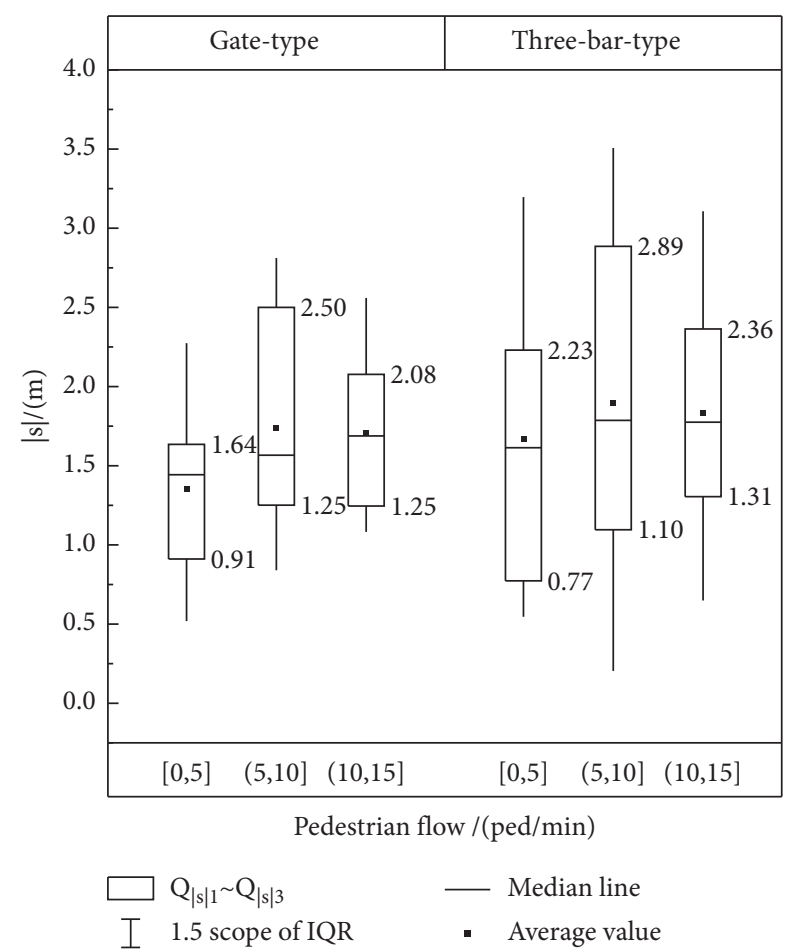

FIgURE 4: The distribution of displacement of pedestrian lane change behavior.

ticket gate facilities, when the pedestrian flow was $[0,5],(5$, $10]$, and $(10,15] \mathrm{ped} / \mathrm{min}$, the threshold $M_{\bar{V}}^{*}$ was $[0.29,1.37]$ $\mathrm{m} / \mathrm{s},(0,1.48] \mathrm{m} / \mathrm{s}$, and $(0,1.95] \mathrm{m} / \mathrm{s}$.

At different types of ticket gate facilities, the average value of average speed $\bar{V}$ was the smallest when the

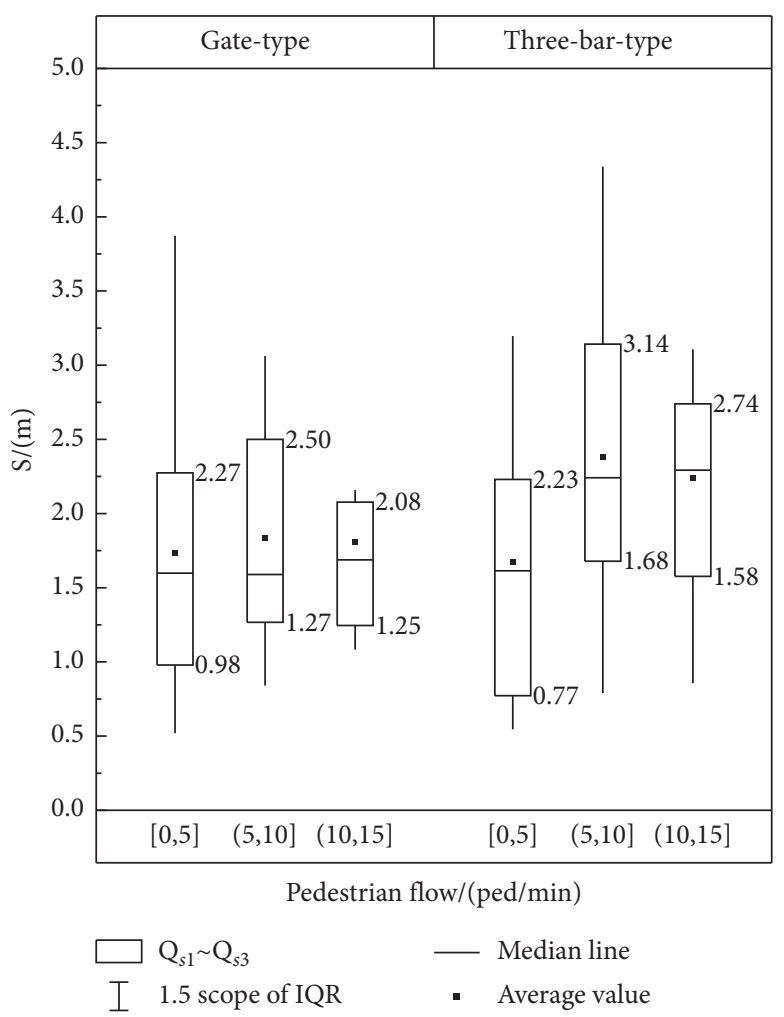

FIgURE 5: The distribution of distance of pedestrian lane change behavior.

pedestrian flow was $(5,10] \mathrm{ped} / \mathrm{min}$. As the pedestrian flow increased, at gate-type ticket gate facilities, the quartile of average speed $\bar{V}$ decreased, and at the 


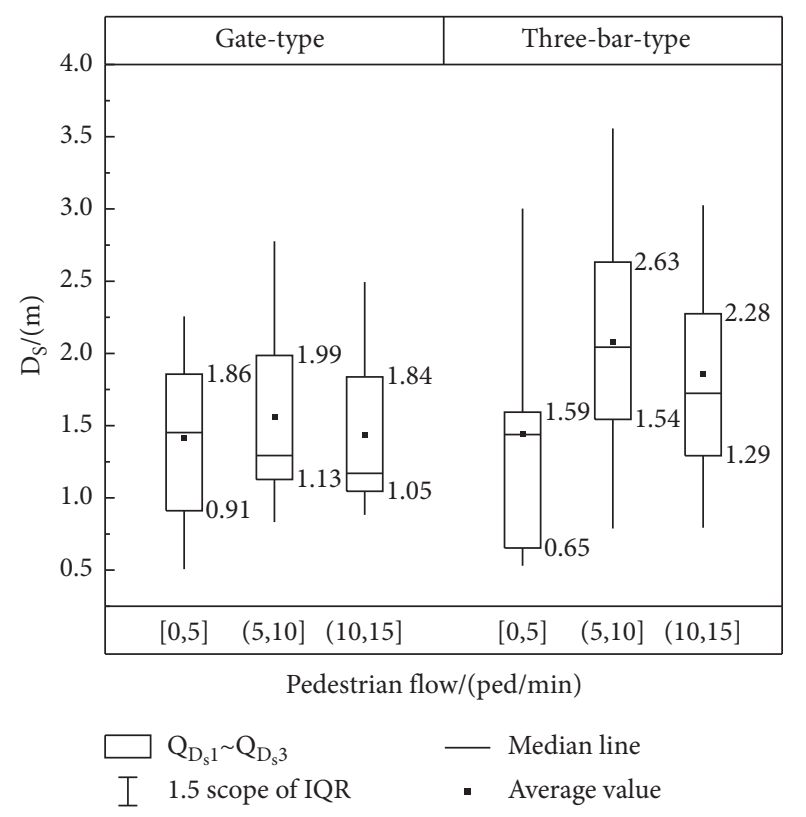

FIgURE 6: The distribution of cumulative side shift distance of pedestrian lane change behavior.

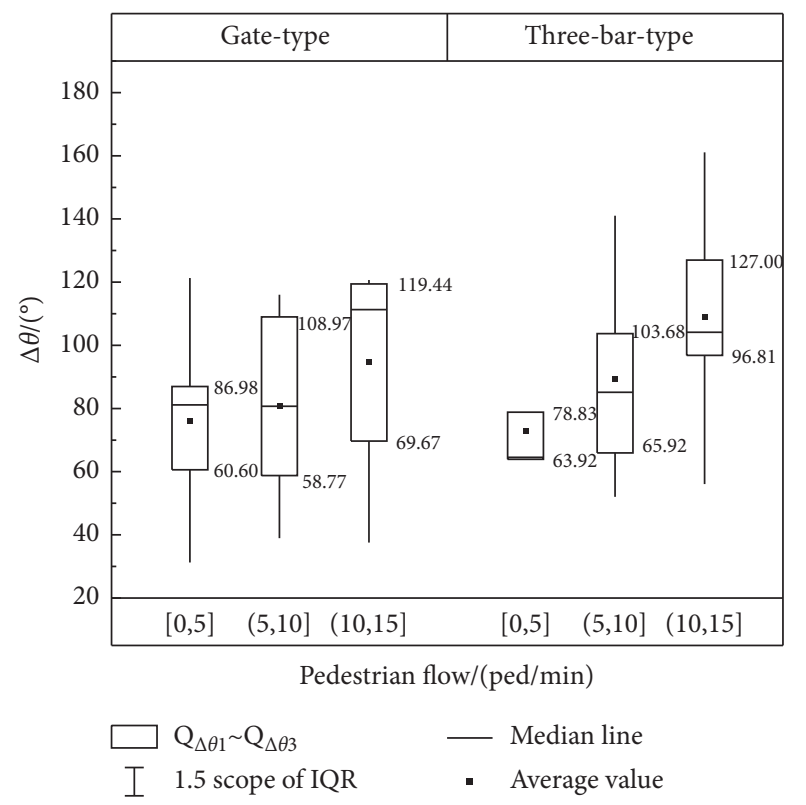

FIGURE 7: The distribution of change in direction of the movement of pedestrian lane change behavior.

pedestrian flow $(10,15]$ person/min, the threshold $M_{\bar{V}}^{*}$ was larger, indicating that pedestrians changed lanes at a faster speed at this time. As the pedestrian flow increased, at the three-bar-type ticket gate facilities, the quartile of average speed $\bar{V}$ increased.

5.1.6. Longitudinal Distance. Figure 9 shows the distribution of longitudinal distance of pedestrian lane change behavior. At gate-type ticket gate facilities, when the pedestrian flow was $[0,5],(5,10]$, and $(10,15] \mathrm{ped} / \mathrm{min}$, the threshold $M_{D_{Y}}^{*}$

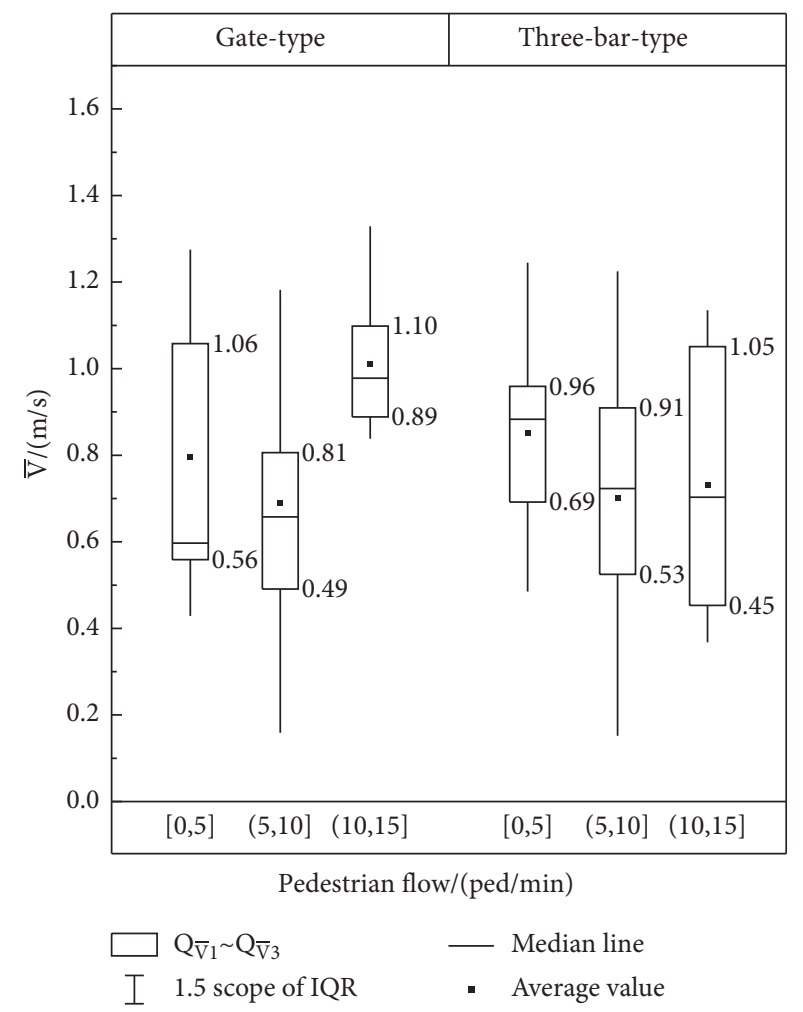

FIGURE 8: The distribution of average speed of pedestrian lane change behavior.

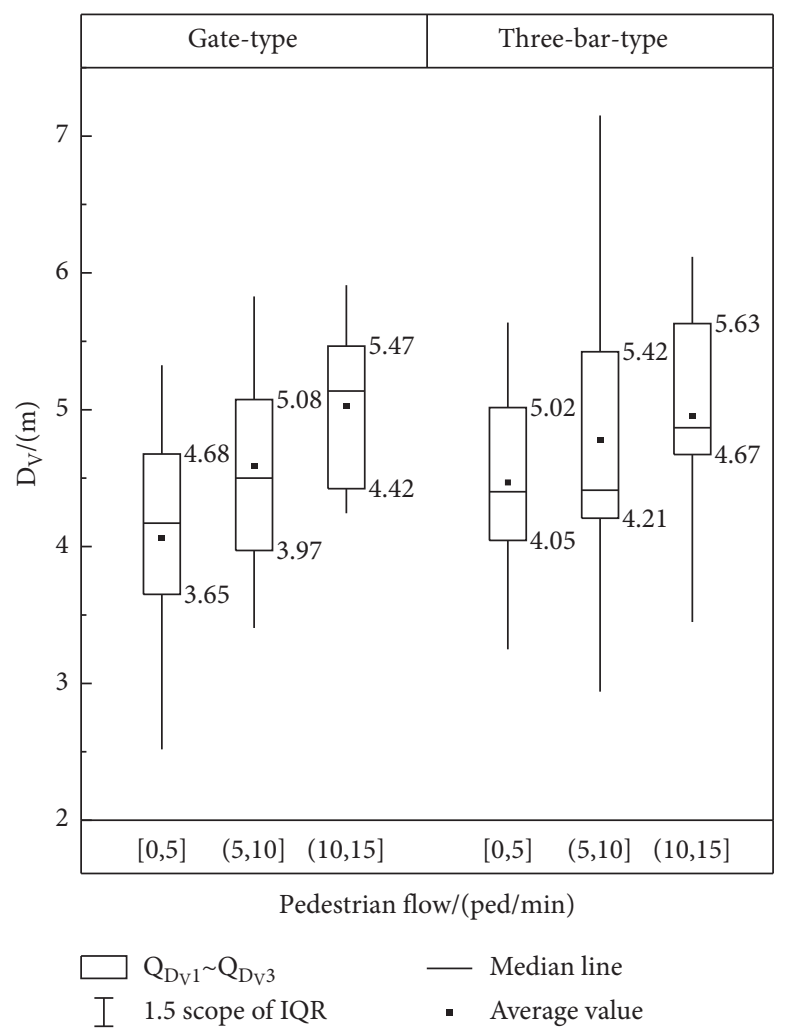

FIGURE 9: The distribution of longitudinal distance of pedestrian lane change behavior. 
TABle 2: Pedestrian lane change behavior spectrum of ticket gate facilities in subway stations.

\begin{tabular}{|c|c|c|c|c|c|c|c|}
\hline & & & ypes of $t$ & te faciliti & & & \\
\hline Ped & flow (ped/min) & & Gate-typ & & & ree-bar- & \\
\hline & & {$[0,5]$} & $(5,10]$ & $(10,15]$ & {$[0,5]$} & $(5,10]$ & $(10,15]$ \\
\hline & Average value & 1.35 & 1.74 & 1.71 & 1.67 & 1.90 & 1.84 \\
\hline$|\vec{s}|(\mathrm{m})$ & Upper limit & 2.74 & 4.38 & 3.33 & 4.42 & 5.58 & 3.94 \\
\hline & Standard deviation & 0.59 & 0.65 & 0.53 & 1.09 & 0.95 & 0.80 \\
\hline & Average value & 1.74 & 1.84 & 1.81 & 1.67 & 2.38 & 2.24 \\
\hline$S(\mathrm{~m})$ & Upper limit & 4.21 & 4.35 & 3.33 & 4.42 & 5.33 & 4.48 \\
\hline & Standard deviation & 1.01 & 0.68 & 0.74 & 1.09 & 0.96 & 1.00 \\
\hline & Average value & 0.80 & 0.69 & 1.01 & 0.85 & 0.70 & 0.73 \\
\hline & Upper limit & 1.81 & 1.29 & 1.42 & 1.37 & 1.48 & 1.95 \\
\hline$V(\mathrm{~m} / \mathrm{s})$ & Lower limit & 0 & 0.01 & 0.58 & 0.29 & 0 & 0 \\
\hline & Standard deviation & 0.34 & 0.31 & 0.17 & 0.29 & 0.28 & 0.30 \\
\hline & Average value & 76.06 & 80.67 & 94.88 & 72.83 & 89.31 & 109.03 \\
\hline$\Delta \theta\left(^{\circ}\right)$ & Upper limit & 126.55 & 184.27 & 194.10 & 101.20 & 160.32 & 172.29 \\
\hline$\Delta \theta()$ & Lower limit & 21.03 & 0 & 0 & 41.56 & 9.28 & 51.53 \\
\hline & Standard deviation & 25.89 & 24.62 & 32.11 & 29.95 & 29.87 & 26.42 \\
\hline & Average value & 4.06 & 4.59 & 5.03 & 4.47 & 4.78 & 4.96 \\
\hline & Upper limit & 6.23 & 6.75 & 7.05 & 6.48 & 7.24 & 7.07 \\
\hline$D_{V}(\mathrm{~m})$ & Lower limit & 2.11 & 2.31 & 2.85 & 2.60 & 2.40 & 3.23 \\
\hline & Standard deviation & 0.87 & 0.85 & 0.62 & 0.91 & 0.97 & 0.81 \\
\hline & Average value & 1.42 & 1.56 & 1.44 & 1.44 & 2.08 & 1.86 \\
\hline$D_{S}(\mathrm{~m})$ & Upper limit & 3.29 & 3.28 & 3.03 & 3.00 & 4.27 & 3.77 \\
\hline & Standard deviation & 0.63 & 0.60 & 0.56 & 0.99 & 0.80 & 0.77 \\
\hline & Average value & 1.11 & 1.11 & 1.00 & 1.00 & 1.21 & 1.31 \\
\hline & Upper limit & 1 & 1 & 1 & 1 & 1 & 1 \\
\hline$k$ (time) & Lower limit & 1 & 1 & 1 & 1 & 1 & 1 \\
\hline & Standard deviation & 0.33 & 0.47 & 0.00 & 0.00 & 0.41 & 0.63 \\
\hline
\end{tabular}

was $[2.11,6.23] \mathrm{m},[2.31,6.75] \mathrm{m}$, and $[2.85,7.05] \mathrm{m}$. At three-bar-type ticket gate facilities, when the pedestrian flow was $[0,5],(5,10]$, and $(10,15] \mathrm{ped} / \mathrm{min}$, the threshold $M_{D_{V}}^{*}$ was $[2.60,6.48] \mathrm{m},[2.40,7.24] \mathrm{m}$, and $[3.23,7.07] \mathrm{m}$.

At different types of ticket gate facilities, as pedestrian flow increased, the average value of longitudinal distance $D_{V}$ increased, and the upper and lower quartiles increased. At the three-bar-type ticket gate facilities, the increase in longitudinal distance $D_{V}$ was small, while longitudinal distance $D_{V}$ at the gate-type ticket gate facilities was greatly affected by the pedestrian flow.

5.1.7. Number of Lane Changes. Under different types of ticket gate facilities and pedestrian flow, the threshold $M_{k}^{*}$ was generally 1 time. Since the purpose of pedestrians was to quickly pass through the gate facilities, the number of gate facilities in the surveyed subway station could meet the needs of pedestrians, so pedestrians basically did not choose to change lanes back and forth.

5.2. Construction of Pedestrian Lane Change Behavior Spectrum of Ticket Gate Facilities in Subway Stations. According to the collected data, the seven indicators of lane change behavior were analyzed, and their average values, thresholds, and fluctuation ranges were obtained, and the pedestrian lane change behavior spectrum of ticket gate facilities in subway stations was constructed, as shown in Table 2.

\section{Discussion}

This research used the pedestrian lane change behavior spectrum to reveal the characteristics of the indicators of pedestrian traffic behaviors under different ticket gate facilities and pedestrian flow conditions, so as to improve the efficiency of pedestrian traffic. We used the constructed behavior spectrum to find that when the pedestrian flow was $(5,10] \mathrm{ped} / \mathrm{min}$, the average values of displacement, distance, and cumulative side shift distance were the largest, the thresholds were the largest, and the number of lane changes was generally once. The longitudinal distance of pedestrians at the gate-type ticket gate facility was greatly affected by the pedestrian flow. The results show that the behavior spectrum can improve the actual capacity of the ticket gate facilities through the selection and scientific layout and can reduce the risk of congestion and collision caused by abnormal lane change behavior by controlling the volatility of indicators of the behavior spectrum.

Lee and Nho [1] used pedestrian-controlled experiments in the university gymnasium and found that the average value of displacement $|\vec{s}|$ was $11.82 \mathrm{~m}$, the average value of distance $S$ was $11.95 \mathrm{~m}$, and the average value of average speed $\bar{V}$ was $1.32 \mathrm{~m} / \mathrm{s}$, which were all larger than those of this research. This was because the two scenarios were different. Pedestrians could travel short distances in front of the ticket gate facilities in subway stations, and the process of preparing electronic equipment or magnetic cards for pedestrians affected the walking speed. Kwon et al. [33] regarded 
telephone poles, parked cars, or parked bicycles on the street as fixed obstacles and found that the average value of the longitudinal distance $D_{V}$ of lane change behaviors from the obstacles was $4.8 \mathrm{~m}$. It was the same as the results of this research, which verified the rationality of the results.

\section{Conclusions}

Based on pedestrian dynamics, this research determined the indicators of pedestrian lane change behavior and used the quartile method to analyze the seven indicators of displacement, distance, average speed, change in direction of the movement, longitudinal distance, cumulative side shift distance, and number of lane changes. Based on the characteristics of three types of factors, namely, types of ticket gate facilities, pedestrian flow, and individual lane change behavior, the pedestrian lane change behavior spectrum of ticket gate facilities in subway stations was constructed, which perfected the theoretical method of the behavior research of ticket gate facilities.

The limitations of the research presented in this paper should be pointed out: tracking pedestrians from surveillance videos of subway stations is the first step in data collection. The pixels of surveillance videos are generally not high, which affects the accuracy of the results. In addition, the influence of pedestrians' age, whether to carry luggage, and other human factors on the threshold of the indicators of lane change behavior spectrum have not yet been considered at present. The next step is to further increase the sample size data of ticket gate facilities and improve the threshold range according to the characteristics of human factors. The improved threshold range can be applied to the ticket gate facilities of the same scene, and the research ideas and methods can also be used to study behavior spectrum characteristics of subway station escalators, platforms, and other scenes. As the recent COVID-19 pandemic has had a significant impact on pedestrian travel characteristics and behavioral characteristics, it is also possible to use behavioral data during the outbreak of a large-scale infectious disease to establish a pedestrian traffic behavior spectrum and analyze the characteristics of the spectrum, and it provides new research ideas for studying the characteristics of virus transmission in subway stations.

\section{Data Availability}

The data used in this research were provided by the National Natural Science Foundation of China at Shanghai University of Engineering Science. The data are available from the authors for academic purposes through e-mail address fangyong87@tongji.edu.cn.

\section{Conflicts of Interest}

The authors declare that they have no conflicts of interest.

\section{Acknowledgments}

This project was supported by the National Natural Science Foundation of China (52072235 and 51608387).

\section{References}

[1] Y. I. Lee and M. S. Nho, "Analysis of obstacle avoidance behavior of pedestrian from field survey using video tracking data," Journal of Transport Research, vol. 18, no. 2, pp. 61-75, 2011.

[2] S. Y. Cheng, L. B. Fu, P. Y. Yang, Y. Liu, and J. Fang, "A modified multi-grid model for studying the effect of obstacle layouts on pedestrian flow in corridors," Fire Safety Science, vol. 28, no. 4, pp. 222-229, 2019.

[3] A. Forde and J. Daniel, "Pedestrian walking speed at unsignalized midblock crosswalk and its impact on urban street segment performance," Journal of Traffic and Transportation Engineering, vol. 8, no. 1, pp. 57-69, 2021.

[4] I. Ištoka Otković, A. Deluka-Tibljaš, S. Šurdonja, and T. Campisi, "Development of models for children-pedestrian crossing speed at signalized crosswalks," Sustainability, vol. 13, no. 2, p. 777, 2021.

[5] S. M. Simmons, J. K. Caird, A. Ta, F. Sterzer, and B. E. Hagel, "Plight of the distracted pedestrian: a research synthesis and meta-analysis of mobile phone use on crossing behaviour," Injury Prevention, vol. 26, no. 2, pp. 170-176, 2020.

[6] Y. Wang, G. Ma, and X. Zhuang, "The effect of cell phone distraction on pedestrians' information processing and behavior during road crossing," Advances in Psychological Science, vol. 29, no. 5, pp. 806-814, 2021.

[7] Z. Kuang, J. Yang, G. L. Li, B. Nan, and L. Zeng, "Pedestrian detection method for station based on improved YOLOv3," Journal of Railway Science and Engineering, vol. 18, no. 1, pp. 55-63, 2021.

[8] J. Li, J. Zhou, Y. Lin, and Q. H. Wang, "Driver behavior during yellow interval based on video detection technology," Journal of Transportation Systems Engineering and Information Technology, vol. 15, no. 1, pp. 69-74, 2015.

[9] F. Orsini, G. Gecchele, M. Gastaldi, and R. Rossi, "Transferability and seasonality in extreme value theory applications to road safety: a case study in an Italian motorway," Advances in Transportation Studies, Special Issue, vol. 2, pp. 33-46, 2020.

[10] G. Tesoriere, T. Campisi, A. Canale, A. Serveino, and F. Arena, "Modelling and simulation of passenger flow distribution at terminal of Catania airport," in Proceedings of the International conference of computational methods in sciences and engineering 2018 (ICCMSE 2018), Thessaloniki, Greece, March 2018.

[11] W. X. Wei, Y. Fang, H. Hu, and H. Ding, "Study on characteristics extraction system of pedestrian traffic behavior for subway stations based on video data mining," Railway Transport and Economy, vol. 43, no. 8, pp. 119-125, 2021.

[12] K. Ismail, T. Sayed, and N. Saunier, "Automated pedestrian safety analysis using video data in the context of scramble phase intersections," in Proceedings of the Annual Conference of the Transportation Association of Canada, Vancover, Canada, October 2009.

[13] S. Basbas, T. Campisi, A. Canale, A. Nikiforiadis, and C. Gruden, "Pedestrian level of service assessment in an area close to an under-construction metro line in Thessaloniki, Greece," Transportation research procedia, vol. 45, pp. 95-102, 2020.

[14] C. Gruden, T. Campisi, A. Canale, G. Tesoriere, and M. Sraml, "A cross-study on video data gathering and microsimulation techniques to estimate pedestrian safety level in a confined space," IOP Conference Series: Materials Science and Engineering, vol. 603, no. 4, Article ID 042008, 2019. 
[15] C. Gruden, I. I. Otković, and M. Šraml, "Neural networks applied to microsimulation: a prediction model for pedestrian crossing time," Sustainability, vol. 12, no. 13, p. 5355, 2020.

[16] W. Daamen and S. Hoogendoorn, "Experimental research of pedestrian walking behavior," Transportation Research Record Journal of the Transportation Research Board, vol. 1828, pp. 20-30, 2003.

[17] D. Wu, Simulation Study on the Pedestrian Behavior in Rail Transport Hub with Agent Technology, Beijing Jiaotong University, Beijing, China, 2013.

[18] W. Y. Shao, Research and Application on Transit Capacity of AFC Facility in Subway Station, Southeast University, Nanjing, China, 2012.

[19] D. F. Zhu, C. Z. Wu, W. H. Chu, and C. Sun, "Research on framework construction of key commercial motor vehicle drivers' behavior spectrum for highway," in Proceedings of the 15th Academic Symposium on Cross-Strait Intelligent Transportation Systems, pp. 10-20, Hsinchu, Taiwan, August 2015.

[20] F. Y. Huang, Y. F. Wu, J. R. Chen, and B. Wu, "Traffic behavior spectrum analysis method based on regional road live data," Journal of Computer Applications, vol. 39, no. 3, pp. 907-912, 2019.

[21] J. R. Chen, Y. F. Wu, and B. Wu, "Driver behavior spectrum analysis method based on vehicle driving data," Journal of Computer Applications, vol. 38, no. 7, pp. 1916-1922+1928, 2018.

[22] J. Lu, K. Wang, and Y. M. Jiang, "Real-time identification method of abnormal road driving behavior based on vehicle driving trajectory," Journal of Traffic and Transportation Engineering, vol. 20, no. 6, pp. 227-235, 2020.

[23] Q. J. He, H. Z. Tu, and H. R. Wei, "Analyzing car-following driving ethogram based on driving simulator," Journal of Wuhan University of Technology, vol. 45, no. 3, pp. 1-11, 2020.

[24] M. Wang, H. Z. Tu, and H. Li, "Prediction of car-following risk status based on car-following behavior spectrum," Journal of Tongji University, vol. 49, no. 6, pp. 843-852, 2021.

[25] X. Chang, H. J. Li, Z. H. Zhang, and Z. F. Huang, "The behavior map of bad vehicle and its application in streamlining lane management in the ramp area," Road Traffic \& Safety, vol. 18, no. 1, pp. 32-37, 2018.

[26] K. Wang, J. Lu, and Y. M. Jiang, "Abnormal road driving behavior spectrum establishment and characteristic value calculation method based on vehicle driving trajectory," Journal of Traffic and Transportation Engineering, vol. 20, no. 6, pp. 236-249, 2020.

[27] G. Y. Zhou, N. Mo, and L. Yang, "On fare gate settings at station halls of Beijing metro," Urban Mass Transit, vol. 9, pp. 83-86, 2013.

[28] Q. J. Wang, Study on Lane Change Behavior Character and its Influence to Traffic Safety, Harbin Institute of Technology, Harbin, China, 2007.

[29] C. Wang, Research on Several Key Problems of Vehicle Lane Change Warning, Chang'an University, Xi'an, China, 2012.

[30] I. Vasconcelos, R. O. Vasconcelos, B. Olivieri, M. Roriz, M. Endler, and M. C. Junior, "Smartphone-based outlier detection: a complex event processing approach for driving behavior detection," Journal of Internet Services and Applications, vol. 8, no. 1, pp. 1-30, 2017.

[31] S. P. Hoogendoorn, W. Daamen, and P. Bovy, Extracting Microscopic Pedestrian Characteristics from Video Data, Annual Meeting of the Transportation Research Board, Washington, DC, USA, 2003.
[32] S. X. Zhou, Research on Algorithm of 3D Track Detecting of Objects in 2D Video, Shanghai Jiaotong University, Shanghai, China, 2010.

[33] Y. Kwon, S. Morichi, and T. Yai, "Analysis of pedestrian behaviour and planning guidelines with mixed street for narrow urban streets," Transportation Research Record Journal of the Transportation Research Board, vol. 1636, pp. 113-123, 1998. 